\title{
Neuroradiological features of lymphomatosis cerebri: A systematic review of the English literature with a new case report
}

\author{
LONG LI $^{1}$, JIA-HUI RONG ${ }^{1}$ and JIE FENG ${ }^{2}$ \\ ${ }^{1}$ Department of Radiology, Guangdong Provincial Corps Hospital of Chinese People's Armed Police Forces, \\ Guangzhou Medical University, Guangzhou, Guangdong 510507; ${ }^{2}$ Diagnostic Imaging Center, \\ Nanfang Hospital, Southern Medical University, Guangzhou, Guangdong 510515, P.R. China
}

Received October 20, 2017; Accepted April 4, 2018

DOI: $10.3892 / \mathrm{ol} .2018 .8839$

\begin{abstract}
Lymphomatosis cerebri is a rare form of diffusely infiltrating primary central nervous system (CNS) lymphoma (PCNSL). The neuroradiological findings of lymphomatosis cerebri have not been adequately characterized, as the relevant literature consists only of case reports and small case series. The present study describes an unusual presentation of lymphomatosis cerebri in a 56-year-old immunocompetent woman who presented with diffusely infiltrating lesions with perivascular curvilinear enhancement on initial magnetic resonance imaging (MRI) and multiple nodules on the later follow-up computed tomography (CT) scan. A systematic review of the literature is also performed searching PubMed between January 1996 and December 2016 to collect all pertinent case reports and series written in the English language with pathologically confirmed lymphomatosis cerebri and diffuse infiltrative PCNSL without cohesive masses on initial
\end{abstract}

Correspondence to: Professor Long Li, Department of Radiology, Guangdong Provincial Corps Hospital of Chinese People's Armed Police Forces, Guangzhou Medical University, 268 Yanling Road, Guangzhou, Guangdong 510507, P.R. China

E-mail: radiolilong@hotmail.com

Abbreviations: ADC, apparent diffusion coefficient; BBB, blood-brain barrier; CNS, central nervous system; CSF, cerebrospinal fluid; CT, computed tomography; DTI, diffusion tensor imaging; DWI, diffusion-weighted imaging; FDG, fluorodeoxyglucose; FLAIR, fluid-attenuated inversion recovery; FOV, field of view; GCB, germinal center B-cell-like; IHC, immunohistochemistry; MRI, magnetic resonance imaging; MRS, magnetic resonance spectroscopy; non-GCB, non-germinal center B-cell type; PCNSL, primary central nervous system lymphoma; PET, positron emission tomography; PRISMA, preferred reporting items of systematic reviews and meta-analyses; SPECT, single-photon emission computed tomography; $\mathrm{T}_{1} \mathrm{WI}, \mathrm{T}_{1}$-weighted image; $\mathrm{T}_{2} \mathrm{WI}, \mathrm{T}_{2}$-weighted image; TE, echo time; TR, repetition time

Key words: lymphomatosis cerebri, primary central nervous system lymphoma, diffuse tumorous infiltration, leukoencephalopathy, neuroradiology, magnetic resonance imaging
MRI. A total of 45 cases were identified from 39 articles and the present case report. The patient ages ranged from 28 to 85 years (mean, 57.3 years). Only 3 patients $(6.7 \%)$ were immunosuppressed (acquired immune deficiency syndrome patients). The most common clinical presentation was cognitive changes or dementia (46.7\%). Cerebrospinal fluid analysis in all cases was non-specific. Diffuse and asymmetric abnormal T2-hyperintensity in deep and subcortical white matter was observed in all cases. Gray matter involvement (17.8\%), spreading along the corticospinal tract (35.6\%) and a slight mass effect (51.1\%) also were observed. Contrast-enhanced patterns on MRI could be divided into three forms of non-enhancement (64.4\%) and non-mass-like enhancement $(35.6 \%)$ on initial MRI, as well as nodular or mass-like enhancement on the later follow-up MRI (15.6\%). There were non-specific findings on magnetic resonance spectroscopy for 4 patients, on positron emission tomography/CT for 12 patients and on single-photon emission CT for 1 patient. Diagnosis was established by brain biopsy in 35 cases (77.8\%) and autopsy in 9 cases (20\%), involving B-cell lymphoma in 40 cases (88.9\%) and T-cell lymphoma in 4 cases (8.9\%). In conclusion, lymphomatosis cerebri, namely diffuse PCNSL or diffuse lymphoma of the CNS, is characterized by rapidly progressive dementia in the elderly, diffusely infiltrated CNS white matter along the corticospinal tract, possible involvement of the gray matter, a slight mass effect and varied contrast-enhancement patterns on MRI. Non-enhancement or non-mass-like enhancement on MRI may be a special form of diffuse PCNL during disease development and progression.

\section{Introduction}

Neuroradiology with cranial magnetic resonance imaging (MRI) using fluid-attenuated inversion recovery (FLAIR) and T1-weighted sequences prior to and following contrast injection combined with diffusion-weighted imaging (DWI) is the method of choice for the diagnosis and follow-up of primary central nervous system (CNS) lymphoma (PCNSL) (1). The typical features of PCNSL are intra-axial homogenous single or multiple contrast-enhancing lesions with markedly restricted diffusion that are in contact with a cerebrospinal fluid (CSF) surface (2).

Lymphomatosis cerebri is a rare form of PCNSL characterized by diffuse lymphomatous parenchymal infiltration 
without evidence of cohesive masses or parenchymal architectural distortion (3). The distinguishing neuroradiological features of lymphomatosis cerebri have not yet been established in detail, as the literature contains only case reports and small series reporting imaging findings. In brain MRI studies, lymphomatosis cerebri can be mistaken for a variety of diffuse infiltrative brain tumors or leukoencephalopathy (2).

The present study first describes a case involving a 56-year-old immunocompetent woman who presented with diffuse infiltrating lesions in the brain parenchyma with perivascular curvilinear enhancement on initial MRI and multiple nodules on the later follow-up computed tomography (CT) scans. The current study also presents the results of a systematic review of the clinical and neuroradiological features of lymphomatosis cerebri. Using strict inclusion criteria, a literature search was performed on the data recorded between January 1996 and December 2016. To the best of our knowledge, this study provides the largest systematic review of neuroradiological features for lymphomatosis cerebri to date, and the present case is the first to be reported with perivascular curvilinear enhancement and subsequent multiple nodules.

\section{Materials and methods}

Case report. Written informed consent was obtained from the daughter of the patient for publication of this case report and any accompanying data. A 56-year-old immunocompetent woman presented Guangdong Provincial Corps Hospital of Chinese People's Armed Police Forces (Guangzhou, China) with acute onset of headache, confusion and inability to relevantly answer questions on February 25, 2015. Blurred vision had occurred 20 days prior to admission. Personality and behavior changes had appeared 10 days prior to admission. The neurological examination showed fluctuating spatiotemporal disorientation with dyscalculia, a weak voice and moderate bradykinesia. Non-contrast brain CT (LightSpeed VCT XT CT 64-row helical CT scanner; GE Healthcare, Chicago, IL, USA) performed on February 25, 2015, showed abnormal low density results in the right parietal white matter, external capsule and thalamus (Fig. 1). On the initial brain MRI (MAGNETOM SKyra 3T MRI scanner; Siemens Healthineers, Erlangen, Germany) performed following admission on February 26, 2015 , the T2-weighted images $\left(\mathrm{T}_{2} \mathrm{WI}\right)$ [repetition time (TR), $6,000 \mathrm{msec}$; echo time (TE), $99 \mathrm{msec}$; slice thickness, $5 \mathrm{~mm}$; distance factor, $30 \%$; voxel size, $0.3 \times 0.3 \times 5.0 \mathrm{~mm}$; field of view (FOV), 22x22 cm] and FLAIR images (TR, 9,000 msec; TE, $85 \mathrm{msec}$; slice thickness, $5 \mathrm{~mm}$; distance. factor, $30 \%$; voxel size, $0.4 \times 0.4 \times 5.0 \mathrm{~mm}$; FOV, $22 \times 22 \mathrm{~cm}$ ) showed asymmetric and diffuse white-matter hyperintensities involving bilateral paraventricular white matter, bilateral temporal lobes, the left occipital lobe, the medial prefrontal cortex, bilateral basal ganglia, the thalamus and the midbrain, as well as a slight mass effect on the posterior horn of the left lateral ventricle (Fig. 2). DWI (TR, 4,500 msec; TE, $64 \mathrm{msec}$; slice thickness, $5 \mathrm{~mm}$; dist. factor, $30 \%$; voxel size, $1.4 \times 1.4 \times 5.0 \mathrm{~mm}$; FOV, $22 \times 22 \mathrm{~cm}$; b value, $1,000 \mathrm{sec} / \mathrm{mm}^{2}$ ) showed mild hyperintense signals, corresponding with T2/FLAIR hyperintensities (Fig. 2). Apparent diffusion coefficient (ADC) maps showed that the areas of increased diffusion signal represented both shine-through artifacts and mild diffusion restriction
(Fig. 2C). Subsequent to the bolus intravenous injection of $10 \mathrm{ml}$ gadodiamide (Omniscan ${ }^{\circledR}$; GE Healthcare), $\mathrm{T}_{1}$-weighted images (T 1 WI) (TR, 250 msec; TE, 2.49 msec; slice thickness, $5 \mathrm{~mm}$; dist. factor, $30 \%$; voxel size, $0.7 \times 0.7 \times 5.0 \mathrm{~mm}$; FOV, $22 \times 22 \mathrm{~cm}$ ) showed that asymmetric perivascular curvilinear enhancement perpendicular to the lateral ventricle was present in the bilateral cerebral white matter, and that eppendymal enhancement around the left ventricle and additional more patchy enhancement in the bilateral cerebral white matter were present (Fig. 2). CSF pressure was $160 \mathrm{mmH}_{2} \mathrm{O}$ during lumbar puncture. CSF anlysis revealed $0.46 \mathrm{~g} / 1$ protein (normal range, 0.15-0.45 g/l), $3.8 \mathrm{mmol} / 1$ glucose (normal range, 3.3-4.4 mmol/l) and $119.8 \mathrm{mmol} / 1$ chloride (normal range, $122-128 \mathrm{mmol} / \mathrm{l}$ ). No bacteria were detected via gram stain and culture in the CSF. Cytological analysis of the CSF was negative for malignancy, and no oligoclonal bands could be found. Autoimmune and viral serologies were negative.

The patient was initially diagnosed with acute disseminated encephalomyelitis and treated with $500 \mathrm{mg}$ methylprednisolone via intravenous infusion once a day for 10 days. Following an improved clinical situation, the patient was discharged on March 15, 2015.

The patient gradually developed gait disturbance, personality changes and dementia beginning on April 10, 2015. On April 22, 2015, a repeat MRI with the aforementioned sequence parameters showed diffuse and asymmetrical pathological signal hyperintensities on T2/FLAIR images, a slightly hyperintense signal on DWI and asymmetric perivascular curvilinear enhancement on $\mathrm{T}_{1} \mathrm{WI}$ with gadolinium that had expanded compared with that in the initial MRI (Fig. 2). Whole-body fluorodeoxyglucose positron emission tomography/CT (FDG-PET/CT) scans (Biograph ${ }^{\mathrm{TM}} \mathrm{mCT}$ PET/CT Scanner; Siemens Healthineers) were also acquired on April 23, 2015 (Fig. 3). No primary FDG-avid malignancy was identified. Brain FDG-PET was markedly abnormal, with increased uptake in the bilateral frontal, parietal, occipital and temporal lobes, basal ganglia, midbrain, cerebellum and cervical cord.

Immunohistochemistry. A stereotactic brain biopsy of the left frontal lobe was performed on May 8, 2015. The tissue specimens were fixed in $10 \%$ neutral buffered formalin solution for $24 \mathrm{~h}$ at room temperature, followed by paraffin embedding. Sections with a thickness of 4 ìm were cut and mounted on glass slides for hematoxylin-eosin (H\&E) staining performed by a 3 min incubation in hematoxylin followed by a $30 \mathrm{sec}$ incubation in eosin at room temperature. For immunohistological (IHC) staining, 4 ìm paraffin tissue sections were mounted on silanized glass slides using chrome-alum gelatin as adhesive. IHC staining was performed by the Streptavidin-Biotin-Complex (SABC) method according to the Sixth Edition of Dako's Educational Guidebook to IHC Staining Methods (4). Sections were deparaffinized in xylene $(2 \times 10 \mathrm{~min})$ and rehydrated through an alcohol gradient: $100 \%$ ethanol $(2 \times 10 \mathrm{~min}), 95 \%$ ethanol $(1 \times 8 \mathrm{~min})$, $80 \%$ ethanol (1x5 min), and 70\% ethanol (1x5 min) followed by $1 \times 10$ min in $\mathrm{ddH}_{2} \mathrm{O}$. Then nonspecific background staining was blocked with Background Sniper (BS966M, Biocare Medical, LLC, Paheco, CA, USA), 3\% bovine serum albumin (BSA; Sigma-Aldrich; Merck KGaA, Darmstadt, Germay) in 

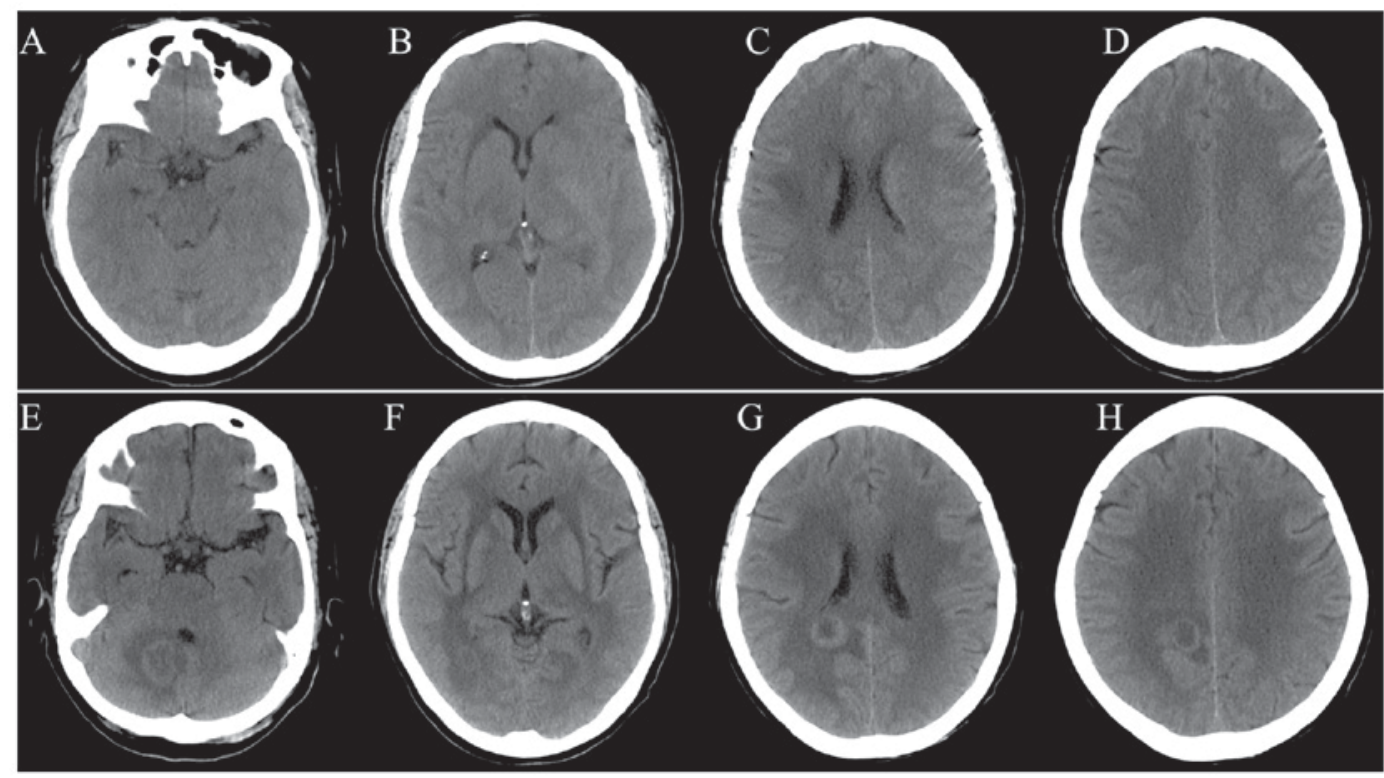

Figure 1. CT findings of lymphomatosis cerebri from the present case report. (A-D) On February 25, 2015, the day of presentation to the neurological clinic, unenhanced CT showed (B) an abnormal low density in the right thalamus, external capsule and (C and D) parietal white matter. (E-H) On July 4, 2015, the follow-up unenhanced CT demonstrated abnormal hyperintensities that extended almost to all cerebral and cerebellar white matter, as well as the thalamus and brainstem. Multiple circular nodules were also observed in (E) the right cerebellar hemisphere, and (F and $\mathrm{G})$ the bilateral occipital white matter and (H) right parietal white matter. CT, computed tomography.
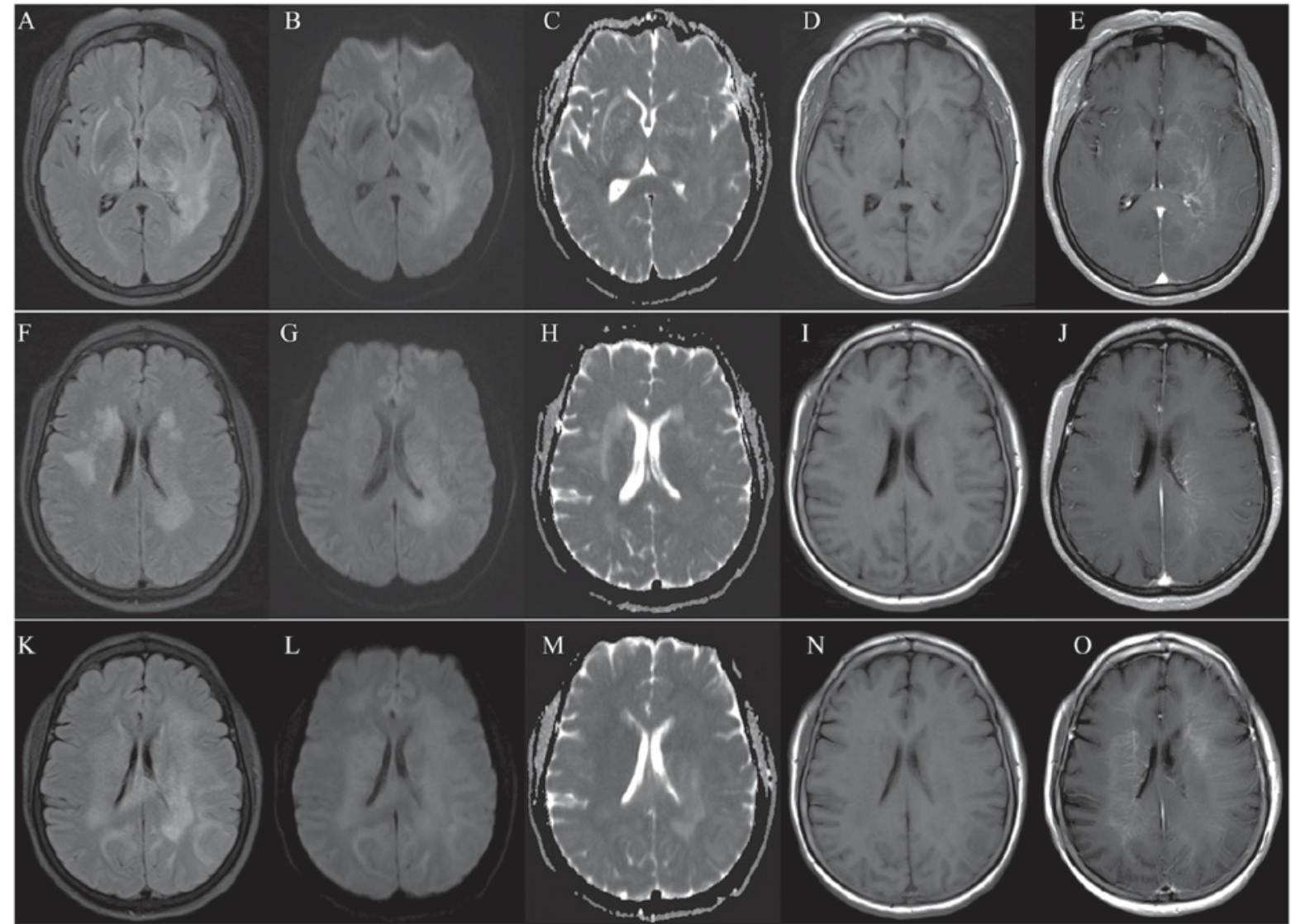

Figure 2. MRI findings of lymphomatosis cerebri from the present case report. (A-J) The initial MRI was performed on February 26, 2015. (A and F) FLAIR and (B and G) DWI showed diffuse multiple asymmetrical hyperintensity in (A) the bilateral basal ganglia and thalamus, (A, B and G) temporal white matter and $(\mathrm{F}$ and $\mathrm{G})$ medial prefrontal cortex and periventricular white matter, as well as a slight mass effect on the posterior horn of the left lateral ventricle. $(\mathrm{C}$ and $\mathrm{H})$ Apparent diffusion coefficient map showed mild hypotensity with no corresponding T2 hyperintensity, denoting a T2 shine-through effect and slight restricted diffusion. The lesions were (D and I) isointense on Tl-weighted spin-echo images, and ( $\mathrm{E}$ and $\mathrm{J}$ ) showed asymmetric perivascular curvilinear enhancement perpendicular to the lateral ventricle following gadolinium administration. (K-O) The follow-up MRI was performed on April 22, 2015. (K) FLAIR and (L) DWI demonstrated broader hyperintensity of the cerebral white matter. (O) The perivascular curvilinear enhancement on $T_{1}$-weighted imaging with gadolinium expanded markedly. MRI, magnetic resonance imaging; FLAIR, fluid-attenuated inversion recovery; DWI, diffusion-weighted imaging. 

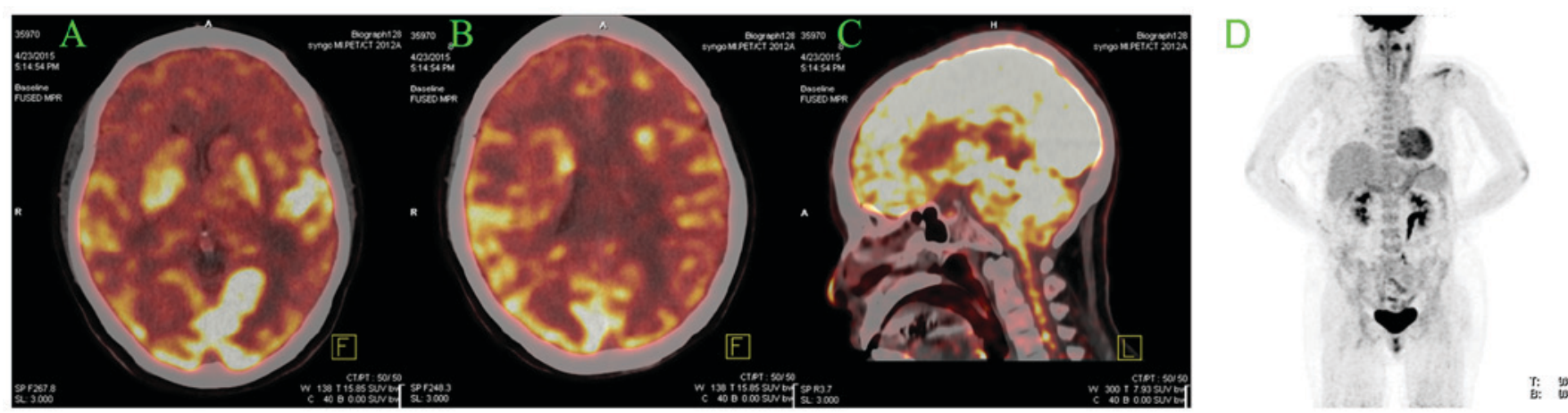

Figure 3. $\left[{ }^{18} \mathrm{~F}\right] \mathrm{FDG}-\mathrm{PET} / \mathrm{CT}$ findings of lymphomatosis cerebri from the present case report. On April 23, 2015, the PET/CT fusion images revealed inhomogeneous and patchy distribution of ${ }^{18} \mathrm{~F}-\mathrm{FDG}$ uptake, with an SUVmax of 20.5 and SUVave of 15.3 in (A and B) the cerebrum, (C) brainstem, (C) cerebellum and (C and D) cervical cord. (D) No sign of systemic lymphoma was present. FDG, fluorodeoxyglucose; PET/CT, positron emission tomography/computed tomography; SUV, standardized uptake value.

phosphate-buffered saline (PBS) and 5\% normal goat-serum solution (OriGene Technologies, Inc., Beijing, China) for $30 \mathrm{~min}$ at room temperature. Slides were incubated overnight at $4^{\circ} \mathrm{C}$ with primary antibodies in $3 \%$ BSA-PBS. The following antibodies were applied as primary antibodies at a 1:1,000 dilution in Bond primary antibody diluent (cat. no. AR9590; Leica Biosystems, Newcastle, UK), Anti-CK (cat. no. 103228N; Ascend Biotechnology Co., Ltd., Guangzhou, China), anti-CD3 (cat. no. 18379501; OriGene Technologies, Inc.), anti-CD20 (cat. no. 104048N; Ascend Biotechnology Co., Ltd., Guangzhou, China), anti-GFAP (cat. no. $112147 \mathrm{~N}$; Ascend Biotechnology Co., Ltd.), anti-S-100 (cat. no. 112117N; Ascend Biotechnology Co., Ltd.), anti-cyclin D1 (cat. no. 17650111; OriGene Technologies, Inc.), anti-CD79a (cat. no. 111027N; Ascend Biotechnology Co., Ltd.), anti-PAX-5 (cat. no. 17600412; OriGene Technologies, Inc.), anti-Bcl-2 (cat. no. 17312610; OriGene Technologies, Inc.), anti-Bcl-6 (cat. no. 18343803; OriGene Technologies, Inc.), anti-CD21 (cat.no.17660809; OriGeneTechnologies,Inc.),anti-Ki-67 (cat. no. 111167N; Ascend Biotechnology Co., Ltd.), anti-CD10 (cat. no. 20045299; Dako; Agilent Technologies GmbH, Waldbronn, Germany), and anti-Mum-1 (cat. no. 102288N; Ascend Biotechnology Co., Ltd.). After rinsing 5 times with $0.05 \%$ Brij-35 in PBS for $1 \mathrm{~min}$, slides were then incubated with the horseradish peroxidase conjugated Goat anti-Human IgG secondary antibody at 1:1,000 dilution (cat. no. 20047066; Dako; Agilent Technologies $\mathrm{GmbH}$ ) for $1 \mathrm{~h}$ at room temperature. The entire section was systematically examined under high power fields (x400 magnification) in Leica DMLA light microscope (Leica Microsystems, Wetzlar, Germany). The criteria for positive staining was defined as $>80 \%$ positive staining cells for the Ki-67 and Mum- 1 antibodies, and $\geq 30 \%$ for the other antibodies including CD10, CD20, CD79a and Bcl- 6 antibodies. The pathological diagnosis was diffuse large B-cell lymphoma of non-germinal center B-cell type (non-GCB), IHC exhibited strong staining for B-cell markers CD-20, CD-79a and Ki-67, as well as negative for CD-10 and Bcl-6; however, was positive for MUM-1 (Fig. 4).

Treatment regimen. High-dose methotrexate and rituximab were administered as follows: $8 \mathrm{~g} / \mathrm{m}^{2}$ methotrexate (day 1) and $375 \mathrm{mg} / \mathrm{m}^{2}$ rituximab (day 3), repeated every 14 days. Following three courses of chemotherapy, severe myelosuppression developed. On July 4, 2015, the patient suddenly collapsed and began comatose, and a non-contrast brain CT showed extension of the pathological hypodensities and multiple circular nodules in the white matter lesions of the cerebrum and cerebellum (Fig. 1). The patient succumbed to severe pulmonary infection and serious bleeding resulting from myelosuppression on September 2, 2015.

Methods. The PubMed database was searched on December 2016 using the following terms: 'Lymphomatosis cerebri', and ('PCNSL' or 'brain lymphoma') and ('non-enhancing' or 'diffuse' or 'diffuse infiltrative'). Cited articles of each relevant study were also reviewed for possible relevant articles. Only articles in the English language literature were evaluated.

The definition of lymphomatosis cerebri in the present systematic review was that the T2/FLAIR abnormal hyperintensity involved at least three cerebral lobes or three anatomical regions of the CNS in accordance with the description of gliomatosis cerebri in the 2007 World Health Organization classification of tumors of the CNS (5), but also that the non-enhancing lesions or the lesions without nodular or mass enhancement were identified on initial MR images. According to information from full-text articles, the following criteria were used to define inclusion into this systematic literature review: i) The full-text article included at least a $\mathrm{T}_{2} \mathrm{WI}$ or FLAIR image, and a contrast-enhanced $\mathrm{T}_{1} \mathrm{WI}$; ii) the lesions involved at least three cerebral lobes or anatomical regions of the brain; iii) the lesions showed non-enhancement or non-nodular/non-mass-like enhancement on the initial MRI; and iv) the lesions were pathologically confirmed as PCNSL. The exclusion criteria were as follows: i) Concurrent systemic lymphoma; ii) intravascular CNS lymphoma; iii) inconclusive histological data; iv) failure to provide any MR image in the full-text article; v) only providing CT images in the full-text article; vi) prior surgery, radiotherapy, chemotherapy or corticosteroid therapy; and vii) a non-English article. Any case report that did not meet this definition and these criteria was excluded from the present systematic review, even if the author referred to the condition as lymphomatosis cerebri (6-11). The present study followed the Preferred Reporting Items for Systematic Reviews and Meta-Analyses (PRISMA) guidelines (12). 


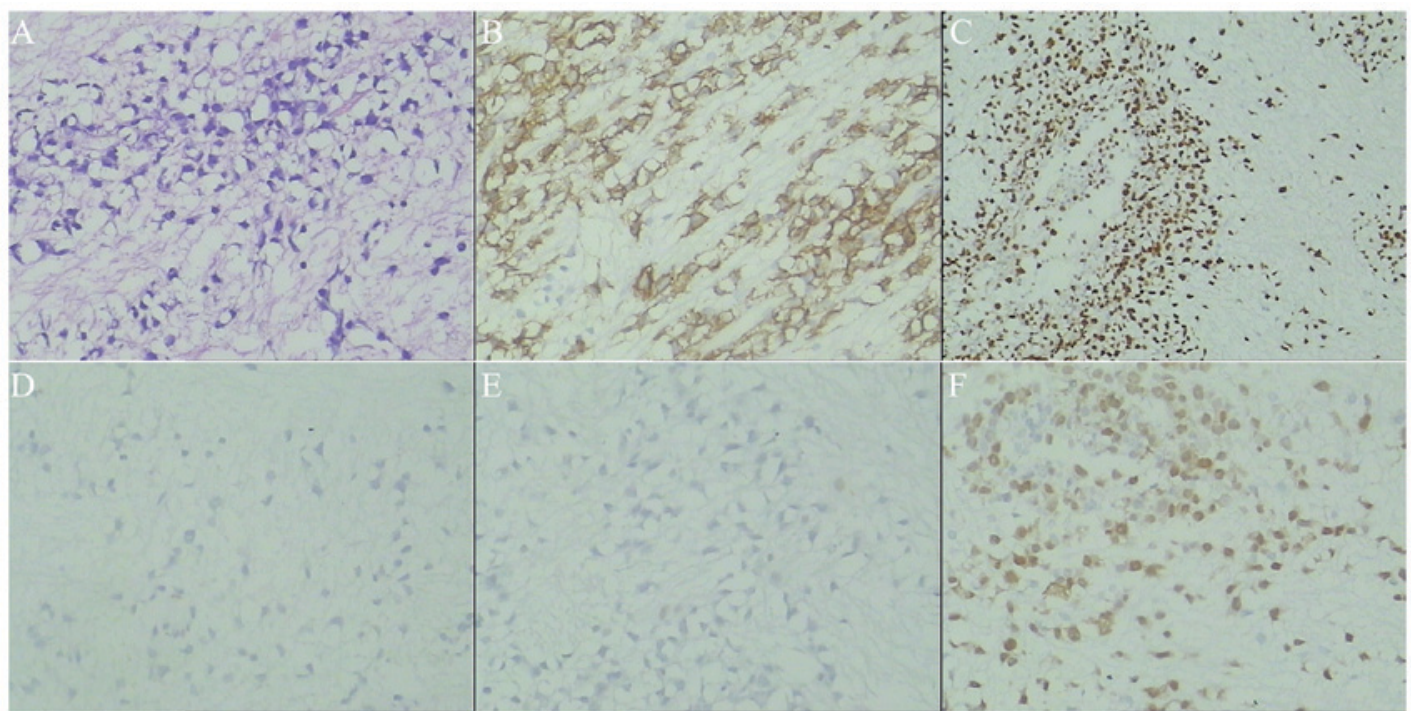

Figure 4. Pathological findings of lymphomatosis cerebri from the current case. On May 12, 2015, a stereotactic brain biopsy of the left frontal lobe revealed (A) prominent diffuse perivascular infiltrates of atypical large B-cells with irregularly shaped nuclei, (B) with strong staining for the B-cell marker CD-20 and for (C) Ki-67, indicative of highly proliferating cells. This case was assigned to the non-germinal center B-cell immunophenotype, as it was negative for (D) CD-10 and (E) Bcl-6, but positive for (F) MUM-1. (A) Hematoxylin and eosin, x400 magnification; (B) anti-CD20, x400 magnification; (C) anti-Ki-67, x200 magnification; (D) anti-CD10, x400 magnification; (E) anti-Bcl-6, x400 magnification; and (F) anti-MUM-1, x400 magnification. CD, cluster of differentiation; Bcl, B-cell lymphoma; MUM-1, multiple myeloma oncogene 1.

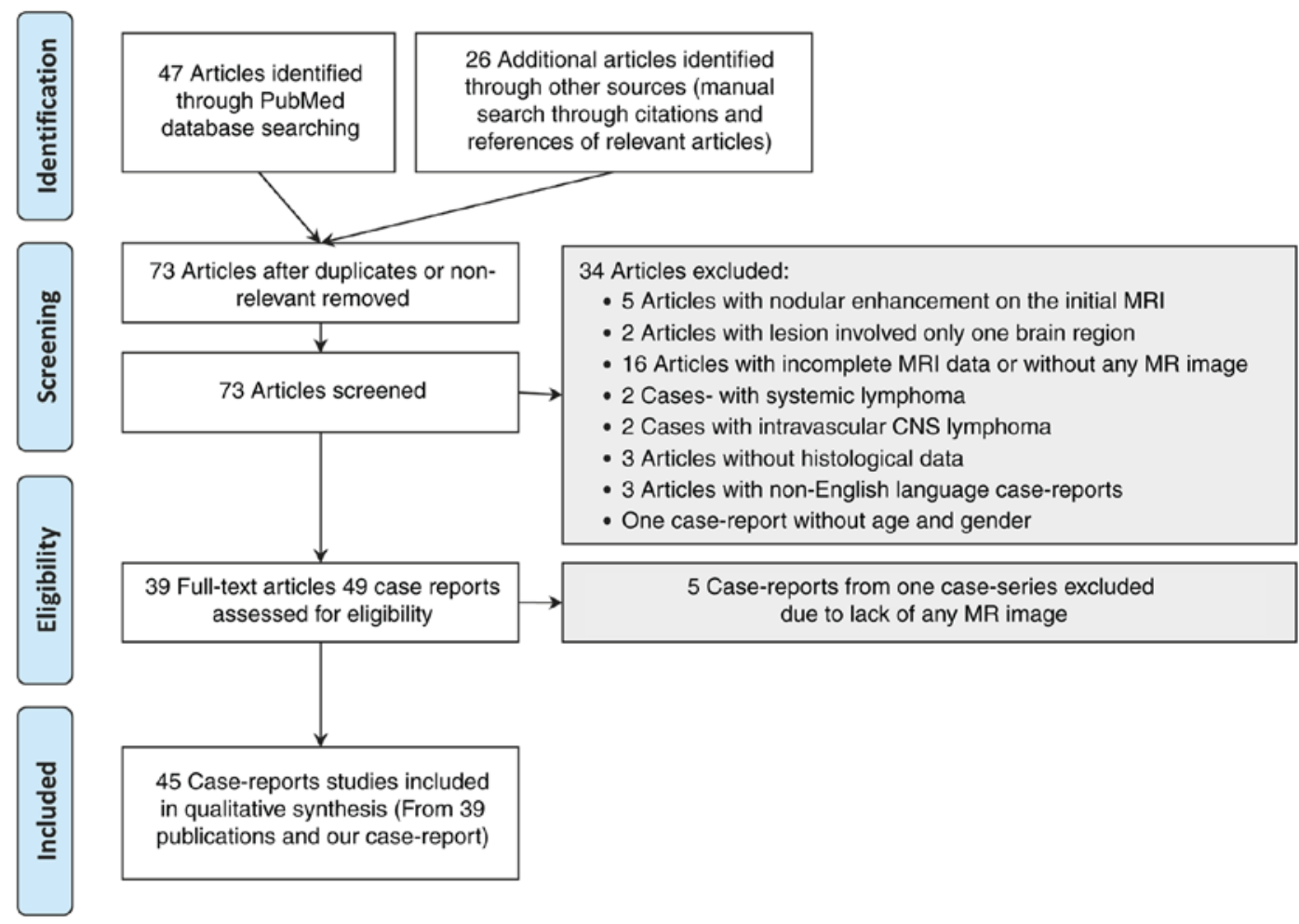

Figure 5. Preferred reporting items of systematic reviews and meta-analyses-style flow diagram of the literature search for the systematic review. MRI, magnetic resonance imaging; CNS, central nervous system.

The collected data included the clinical data, pathological results and imaging findings. Neuroradiological techniques analyzed included CT, MRI [ $\mathrm{T}_{1} \mathrm{WI}, \mathrm{T}_{2} \mathrm{WI}$, FLAIR, $\mathrm{T}_{1} \mathrm{WI}$ with gadolinium contrast enhancement, DWI, diffusion tensor imaging (DTI) and magnetic resonance spectroscopy (MRS)], single-photon emission computed tomography (SPECT) and PET (if any). The lesion location, distribution, morphology, density, contrast enhancement and follow-up imaging were recorded.

\section{Results}

Search results. The PRISMA-style flow diagram of identifying literature for this systematic review is outlined in Fig. 5 . 
Table I. Summary of demographic and clinical features in 45 cases of lymphomatosis cerebri based on a systematic review.

\begin{tabular}{ll}
\hline Characteristic & Value \\
\hline
\end{tabular}

Patients, $\mathrm{n}$

Age, years

Mean

Range

$28-85$

Sex, $\mathrm{n}(\%)$

Male

$23(51.1)$

Female

Clinical symptoms, $\mathrm{n}(\%)$

Cognitive changes/dementia

Gait ataxia

Motor deficit

Sensory deficit

Language deficit

Visual problems

Memory loss

Personality changes

Seizures

Changes in consciousness

Nausea or vomiting

Headache

Persistent fever

Immune status, n (\%)

Immunocompetent

Immunocompromised (AIDS patients)

CSF examination ${ }^{\mathrm{a}}$, n (\%)

Normal

Abnormal

Elevated protein

Increased cell counts

Decreased glucose

Lymphocytosis

Atypical lymphocytes

Pathology sampling, n (\%)

Brain biopsy

Autopsy

Not mentioned

$9(20.0)$

$1(2.2)$

Results

B-cell type

$40(88.9)$

T-cell type

Unclassified

Outcome, months after symptom onset

Succumbed

Alive

$16(35.6)$

5 (11.1)

$11(24.4)$

5 (11.1)

$11(24.4)$

8 (17.8)

4 (8.9)

$10(22.2)$

1 (2.2)

4 (8.9)

2 (4.4)

42 (93.3)

3 (6.7)

5 (14.3)

30 (85.7)

22

12

4

1

1

35 (77.8)
PubMed database searches and reference articles searched by manual methods identified 73 articles. A total of 34 articles were excluded that did not meet the review inclusion criteria. Furthermore, 5 case reports from 1 case series were excluded due to a lack of any MR image. Thus, 39 relevant publications were included in the present systematic review for a total of 44 previously reported cases (13-51). All publications in this review were case studies without controls, and their publication spanned between January 1996 and December 2016. With the addition of the present new case, the total study cohort was 45 cases of lymphomatosis cerebri. The clinical and neuroradiological features in these 45 cases of lymphomatosis cerebri from the systematic review of the literature are summarized in Tables I and II. A list of detailed clinical data and neuroradiological findings collected from all available case reports are shown as 'On-line Table I' and 'On-line Table II' in the online repository Baidu Netdisk (https://pan. baidu.com/s/1KeHH0w0aBR6vKO_mLb2lPA).

Clinical data. The mean age at onset was 57.3 years (range, 28-85 years), including 22 females and 23 males (sex ratio, 1:1.05). A total of 3 patients (6.7\%) were immunosuppressed as a result of acquired immunodeficiency syndrome.

The most common symptoms at onset were progressive dementia and cognitive deficit (44.4\%), gait ataxia $(37.8 \%)$, motor deficit (35.6\%), memory loss (24.4\%), language deficit $(24.4 \%)$, changes in consciousness $(22.2 \%)$, personality changes $(17.8 \%)$, visual problems $(11.1 \%)$ and sensory deficit $(11.1 \%)$. Other clinical features included headaches $(8.9 \%)$, seizures $(8.9 \%)$, persistent fever $(4.4 \%)$, nausea or vomiting $(2.2 \%)$, anorexia $(2.2 \%)$, hypersomnia $(2.2 \%)$, fatigue $(2.2 \%)$ and vertigo $(2.2 \%)$.

CSF analysis was mentioned in 35 cases. Abnormal results were observed in 30 of these cases (85.7\%), including elevated protein in 22 cases, increased cell counts in 12 cases, decreased glucose in 4 cases, lymphocytosis in 1 case and atypical lymphocytes in 1 case (Table I). None of the $35 \mathrm{CSF}$ cytology reports revealed neoplastic lymphocytes.

Neuroradiology. The most common localization of diffuse and asymmetric abnormal T2 hyperintensity involved the cerebral white matter in at least three cerebral lobes or three anatomical regions of the CNS on initial MRI matter (35 cases, $77.8 \%$ ). The lesions most commonly affected the deep and periventricular white matter, and predominantly involved white matter regions of the frontal, parietal, occipital and temporal lobes. Subcortical white matter hyperintensity and U-fiber involvement were frequently observed (24 cases, $53.3 \%$; including the present case) $(13-15,17-19,22,25,26,29,32$, $34,36,38,40,42,43,45,48-50)$. The lesions involved the corpus callosum in 19 cases (42.2\%), the basal ganglia in 15 cases $(33.3 \%)$, the thalamus in 12 cases $(26.7 \%)$, the midbrain in 21 cases $(46.7 \%)$, the pons in 20 cases $(44.5 \%)$, the medulla in 14 cases $(31.1 \%)$, the cerebellum in 12 cases $(26.6 \%)$ and the spinal cord in 5 cases $(11.1 \%$ ) (Table II). Lesions were also observed in the cerebral $(20,28,41,43,50)$ and cerebellar $(35,41)$ cortices, and the cerebellar dentate nuclei (30).

The lesion signals on $\mathrm{T}_{2} \mathrm{WI} / \mathrm{FLAIR}$ MRI were of high intensity in all cases (13-51). Non-enhanced $\mathrm{T}_{1} \mathrm{WI}$ as used for 13 patients in the systematic review, of which 6 cases were

${ }^{a} n=35$. AIDS, acquired immunodeficiency syndrome; CSF, cerebrospinal fluid. 
Table II. Summary of neuroimaging features in 45 cases of lymphomatosis cerebri based on a systematic review.

\begin{tabular}{|c|c|}
\hline Characteristic & Value $(\%)$ \\
\hline \multicolumn{2}{|l|}{ MRI (Initial) } \\
\hline \multicolumn{2}{|l|}{ Localization $(n=45)$} \\
\hline \multicolumn{2}{|l|}{ Cerebrum } \\
\hline \multirow[t]{2}{*}{ Frontal lobe } & 35 (77.8): Unilateral 8 (17.8), \\
\hline & Bilateral 27 (60.0) \\
\hline \multirow[t]{2}{*}{ Parietal lobe } & 32 (73.3): Unilateral 6 (13.3), \\
\hline & Bilateral $26(57.8)$ \\
\hline \multirow[t]{2}{*}{ Temporal lobe } & 33 (73.4): Unilateral 7 (15.6), \\
\hline & Bilateral 26 (57.8) \\
\hline \multirow[t]{2}{*}{ Occipital lobe } & 29 (64.4): Unilateral 5 (11.1), \\
\hline & Bilateral 24 (53.3) \\
\hline \multirow[t]{2}{*}{ Insular lobe } & 26 (57.8): Unilateral 4 (8.9), \\
\hline & Bilateral $22(48.9)$ \\
\hline Periventricular & 10 (22.2): Unilateral 2 (4.4), \\
\hline white matter & Bilateral 8 (17.8) \\
\hline Corpus callosum & $19(42.2)$ \\
\hline \multirow[t]{2}{*}{ Basal ganglia } & 15 (33.3): Unilateral 1 (2.2), \\
\hline & Bilateral 14 (31.1) \\
\hline \multirow[t]{2}{*}{ Thalamus } & 12 (26.7): Unilateral 4 (8.9), \\
\hline & Bilateral 8 (17.8) \\
\hline \multicolumn{2}{|l|}{ Brainstem } \\
\hline \multirow[t]{2}{*}{ Midbrain } & 21 (46.7): Unilateral 3 (6.7), \\
\hline & Bilateral $18(40.0)$ \\
\hline \multirow[t]{2}{*}{ Pons } & 20 (44.5): Unilateral 3 (6.7), \\
\hline & Bilateral 17 (37.8) \\
\hline \multirow[t]{2}{*}{ Medulla } & 14 (31.1): Unilateral 3 (6.7), \\
\hline & Bilateral $11(24.4)$ \\
\hline \multirow[t]{2}{*}{ Cerebellum } & 12 (26.6): Unilateral 1 (2.2), \\
\hline & Bilateral 11 (24.4) \\
\hline Spinal cord & $5(11.1)$ \\
\hline \multicolumn{2}{|l|}{ Signal } \\
\hline \multicolumn{2}{|l|}{$\mathrm{T}_{1} \mathrm{WI}(\mathrm{n}=13)$} \\
\hline Isointensity & $6(46.2)$ \\
\hline Hypointensity & $7(53.8)$ \\
\hline $\begin{array}{l}\text { T2/FLAIR }(n=45), \\
\text { hyperintensity }\end{array}$ & $45(100.0)$ \\
\hline \multicolumn{2}{|l|}{ DWI (n=14) } \\
\hline Isointensity & $2(14.3)$ \\
\hline Slightly hyperintensity & $11(78.6)$ \\
\hline High hyperintense & $1(7.1)$ \\
\hline \multicolumn{2}{|l|}{ Enhancement $(\mathrm{n}=45)$} \\
\hline Absence & $29(64.4)$ \\
\hline Non-mass-like enhancement & $16(35.6)$ \\
\hline Subtle (faint/slight/ & $7(43.8)$ \\
\hline \multicolumn{2}{|l|}{ little/minimal) } \\
\hline Patchy & $5(31.2)$ \\
\hline Perivascular curvilinear & $4(25.0)$ \\
\hline Slight mass effect & $23(51.1)$ \\
\hline $\operatorname{MRS}(n=4)$ & \\
\hline
\end{tabular}

Table II. Continued.

\begin{tabular}{lc}
\hline Characteristic & Value (\%) \\
\hline N-acetylaspartate peak & $1(25.0)$ \\
Normal & $3(75.0)$ \\
Decreased & \\
Choline peak & $1(25.0)$ \\
Normal & $3(75.0)$ \\
Increased & $1(25.0)$ \\
Myo-inositol peak, increased & $1(25.0)$ \\
Lipid peak, increased & $1(25.0)$ \\
Creatine peak, normal & \\
SPECT (n=1) & $1(100.0)$ \\
Increased uptake & \\
PET/CT (n=12) & $4(33.3)$ \\
Increased uptake & $3(25.0)$ \\
Decreased uptake & $5(41.7)$ \\
Normal metabolism &
\end{tabular}

MRI, magnetic resonance imaging; $\mathrm{T}_{1} \mathrm{WI}, \mathrm{T}_{1}$-weighted imaging; FLAIR, fluid-attenuated inversion recovery; DWI, diffusion-weighted imaging; MRS, magnetic resonance spectroscopy; SPECT, single-photon emission computed tomography; PET/CT, positron emission tomography/computed tomography.

presented as isointensity and 7 cases were hypointensity. DWI results were described for 14 patients in the present systematic review, of which the lesion signals were isointense in 2 cases $(34,39)$, slightly hyperintense in 11 cases (including the present case) $(14,20,26,28,29,32,47,48,50)$ and highly hyperintense in 1 case (24) compared with the normal gray matter.

A slight mass effect could have been caused by abnormal T2 hyperintensities, and consisted of mild compression of the adjacent ventricle or cistern in 18 cases (including the present case) $(17,18,20,22,23,28,30-33,40,41,44-48)$ or mild sulcal effacement in 5 cases $(21,36,40,41,49)$.

Follow-up CT and/or MRI were mentioned or described for 31 patients. Among them, 10 cases reported 3 brain MRI examinations. In the second MRI, 24 cases showed progression (including the present case) $(13,14,17,19,20,23,25-27$, 30,33,35,37,38,41-47,49-51) (Figs. 2 and 3), 5 cases demonstrated reduction of lesions $(28,32,36,37,39)$ and 2 cases showed no change from the initial scan $(26,34)$. In the third MRI, when reported, 6 cases showed the enlargement of lesions $(32,35,41,46,49)$ and 4 cases revealed the reduction of lesions $(23,26,30,34,37)$. In 16 cases, lesion extension was described as spreading along the corticospinal tract down to the basal ganglia, thalamus, midbrain, pons, medulla oblongata and the left cerebellar hemisphere, even into the spinal cord. Lesions extending through the corpus callosum were also observed $(23,25,37,39,40)$.

Contrast enhancement patterns on MRI could be divided into three forms: i) Non-enhancing (64.4\%) $(13-24,26-28,30,32,33,37,38,42,43,45,46,48-51)$ on initial MRI; ii) non-mass-like enhancement $(35.6 \%)$ on initial MRI; and iii) nodular or mass-like enhancement on follow-up MRI 
(15.6\%). The non-mass-like enhancements on initial MRI were described as subtle (subtle, faint, slight, little and minimal) in 7 cases $(34-36,39,40,44,47)$, patchy in 5 cases $(22,29,32,48)$ and curvilinear in 4 cases (including the present case) $(25,37,41)$ (Fig. 4). In 8 cases with a non-enhancing pattern on initial MRI, follow-up contrast enhancement MRI revealed patchy enhancement in 5 cases $(30,35,42,43,47)$, single nodular enhancement in 1 case (37) and mass-like enhancement in 2 cases $(35,50)$. In 1 case that presented as a slight enhancement on initial MRI, mass-like enhancement was subsequently found at 4 months post-admission (35). In 4 cases with perivascular curvilinear enhancement on initial MRI, follow-up contrast enhancement MRI revealed patchy enhancement at 5 months post-admission in 1 case, multiple nodular enhancement in 2 cases (including the present case) (25) and multiple mass-like enhancement in 1 case (41).

MRS findings were described for 4 patients. MRS revealed low $\mathrm{N}$-acetylaspartate levels and high choline peaks in 3 cases $(28,29,51)$, with low myo-inositol peaks and high lipid peaks in 1 of these cases (51). The remaining case showed normal concentration patterns of choline, creatine and $\mathrm{N}$-acetyl aspartate (49).

${ }^{18} \mathrm{~F}-\mathrm{FDG} \mathrm{PET} / \mathrm{CT}$ findings were mentioned in 12 patients, including 4 cases that revealed high uptake (including the present case) $(25,47,50)$ (Fig. 4), 3 cases with decreased uptake $(31,36,47)$ and 5 cases with normal metabolism $(23,26,29,34,43)$.

SPECT findings were described in only 1case, and revealed hyperperfusion (49).

Pathology. All the studies confirmed the diagnosis as lymphomatosis cerebri, by pathological analysis of a brain biopsy in 35 cases (including the present case) $(13,14,16,17,19-23,25$, $26,28-30,32,34-38,40-42,44-50)$ or autopsy in 9 cases $(15,17,18$, $24,27,31,33,39,43)$. Only 1 case did not describe the method of pathological sampling (44). Tumor cells were distributed throughout the white matter. Single dispersed round neoplastic cells with prominent nuclei were identified under high-power magnification. The typical neoplastic cells were neither cohesive nor formed an ill-defined tumor mass. No necrosis was present. Overall, three patterns of neoplastic cell infiltration were observed in three forms: i) Diffuse scattered infiltration (including the present case) $(13,15,17,19,22,36-38$, 40,41,47,48) (Fig. 5); ii) perivascular distribution (including the present case) $(15,16,18,29,33-35,38,39,45,48,49)$ (Fig. 5); and iii) massive clusters $(27,32,42,45)$.

A total of 40 cases $(88.9 \%)$ were classified immunohistochemically as B-cell lymphoma and 4 cases $(8.9 \%)$ were T-cell lymphoma $(15,28,29,36)$, while 1 case $(2.2 \%)$ was not further classified (18).

Diffuse large B-cell lymphomas were divided into non-GCB in 5 cases (including the present case) $(26,30,47,48)$ (Fig. 4) and GCB in 1 case (31).

Treatment and prognosis. Overall, 53.3\% (24/45) of cases, including the present case, were treated with steroids prior to the diagnosis of lymphomatosis cerebri. Following the stereotactic brain biopsy diagnosing lymphomatosis cerebri, $22.2 \%(10 / 45)$ of cases were treated with radiotherapy. Treatment with chemotherapy occurred in $31.1 \%$ (14/45) of cases. Even with aggressive treatment with steroids, radiotherapy and chemotherapy, the median survival time was $<24$ months.

\section{Discussion}

An understanding of the neuroradiological characteristics of lymphomatosis cerebri has yet to be resolved. The histopathological pattern of diffusely infiltrating microgliomatosis (PCNSL) was first described by the case report of Fisher et al (52) in 1969. Gross pathological sections of primary reticulum cell sarcoma of the brain (PCNSL) involved diffusely in the white matter were shown by Schaumburg et al (53) in 1972. The CT finding of a diffuse hypodense lesion without mass effect and contrast enhancement in widely infiltrating PCNSL that was proven pathologically by the brain biopsy was described by the case report of Tadmor et al (54) in 1978. MRI features of primary brain T-lymphoma presenting as progressive leukoencephalopathy were reported by the case report of Provinciali et al (55) in 1988, but it was not included in the present systematic review due to the lack of contrast-enhanced $\mathrm{T}_{1} \mathrm{WI}$. The diffusely infiltrating PCNSL without formation of well-defined tumor masses was referred to as 'lymphomatosis cerebri' by the case report of Bakshi et al (17) in 1999, on the analogy of the term 'cerebral gliomas'. For a long time, the literature contained only case reports and small series regarding the clinical and radiological features of lymphomatosis cerebri. Moreover, the term 'lymphomatosis cerebri' has not been widely accepted, so the literature has a variety of descriptions for this diffusely infiltrating PCNSL. The recently published systematic review by Izquierdo et al (48) was the most detailed and the largest study on the clinical characteristics of 42 cases with lymphomatosis cerebri; however, the present systematic review focused on neuroradiological features of lymphomatosis cerebri using inclusion and exclusion criteria that differed from those used by Izquierdo et al (48). The present study defined lymphomatosis cerebri as a special entity with abnormal T2 hyperintensities that involved at least three cerebral lobes or three anatomical regions of the CNS, and where the non-enhancing lesions or the lesions without nodular or mass enhancement were identified on initial MR images. By contrast, the study by Izquierdo et al (48) represented lymphomatosis cerebri as the presence of diffuse white-matter disease in the brain MRI without contrast enhancement (although patchy contrast enhancement was also allowed).

The most common clinical presentation in the present systematic review was cognitive change or dementia (46.7\%). Symptoms include persistent headache, seizures, nausea or vomiting, which all occur in other primary brain tumors (56), but appear less commonly in lymphomatosis cerebri. Lymphomatosis cerebri is observed most commonly in the aged adult, and tumor development appears to be unassociated with the immune status of the individuals. Conventional CSF analysis is non-specific. This indicates that lymphomatosis cerebri may rarely involve the leptomeninges. If CSF cytology and flow cytometry have negative results, definitive diagnostic methods should be considered rather than multiple lumbar punctures. With regard to the survival prognostic factors, the most recently published systematic review has suggested that Karnofsky Performance Status scores $\geq 70$ and treatment with methotrexate 
are independently associated with a favorable outcome, whereas the type of T-cell lymphoma is an independent adverse prognostic factor in patients with lymphomatosis cerebri (48).

Diffuse abnormal T2 hyperintensity is one of the common characteristics of lymphomatosis cerebri. This abnormal T2-hyperintensity can involve the whole CNS, including the brain and spinal cord. A prominent feature of abnormal T2-hyperintensity is the involvement of the deep and periventricular white matter, and involvement of every lobe is reported. Subcortical white matter hyperintensity and U-fiber involvement are frequently observed. Corpus callosum and gray matter involvement are also apparent. The lesion distribution appears to have the propensity for diffuse infiltrative invasion throughout the CNS. It would be more precise if 'lymphomatosis cerebri' were renamed 'diffuse PCNSL' or 'diffuse lymphoma of the CNS'.

The spread of lesions along the corticospinal tract is another common characteristic of diffuse PCNSL based on the results in the present review. In 16 cases reporting follow-up MRI, extensions of lesions were clearly described, spreading along the corticospinal tract down from the cerebral white matter to the spinal cord. The case reported by Samani et al (42) is a representative example. The case reported by Chen and Dong (46) exhibited lesions spreading along the corticospinal tract in upwards and downwards directions at the same time. Lesions extending through the corpus callosum can also be found $(30,42,44,46,47)$. The distribution and spread pattern of this diffuse abnormal signal mimics acute disseminated encephalomyelitis (57).

Contrast enhancement patterns of diffuse PCNSL on MR images could be divided into three forms: i) Non-enhancement; ii) various shapes of non-mass-like enhancement on initial MR images; and iii) single or multiple nodular or mass-like enhancement on the later follow-up MRI. Perivascular curvilinear enhancement in the present case appears to be first reported for diffuse infiltrative PCNL. Actually, this perivascular curvilinear enhancement was first described by Alsherbini et al (58) in 2014, in an immune-competent patient who presented with diffusely infiltrating PCNL involving the brainstem. In addition, 5 cases diagnosed definitively as PCNL presented as single or multiple nodular or mass-like enhancement with diffuse leukoencephalopathy on initial MR images, and then became diffusely infiltrating lesions on follow-up (9,55,59-61). Therefore, we speculate that these contrast enhancement patterns could occur at various stages during the development and progression of the disease, and that non-enhancement or non-mass-like enhancement on initial MRI may be only a special pattern of diffuse infiltrative PCNL.

On the basis of the aforementioned neuroimaging findings, similarly to gliomatosis cerebri (5), we hypothesize that diffuse PCNSL could be divided into primary and secondary subtypes, with primary subtypes exhibiting extensive CNS involvement at the time of initial clinical presentation, and secondary subtypes consisting of progressive infiltration of the brain by a typical locally infiltrative lymphoma observed on clinical follow-up over time. Primary subtypes could then be further divided into type 1 (classical form) and 2, with the classical form presenting as diffuse infiltration without a tumor mass at initial clinical presentation, and type 2 presenting as extensive CNS involvement and a tumor mass.
Radiological-pathological correlation of these contrast enhancement patterns in diffuse PCNSL has not previously been described in detail. It is generally recognized that the patterns and mechanisms of contrast-enhancement depend mostly on the permeability of the blood-brain barrier (BBB) and the extent of vascularity in CNS lesions (62), as well as tumor cellularity (63). The permeability of the BBB is the dominant factor in determining contrast enhancement patterns. The intact BBB will prevent the leakage of contrast material. In the regions with varying degrees of impairment of the BBB, the contrast agent will leak across the vessel wall and begin to accumulate in the perivascular interstitial fluid, which leads to interstitial (extravascular) enhancement $(62,63)$. The non-enhancement pattern is hypothesized to occur when tumor cells are diffusely infiltrative and have not resulted in significant disruption of the BBB $(13,14,16,18,20,26,30,31)$. Another possibility is the vanishing cancer phenomenon caused by corticosteroid-induced apoptosis and lymphoma cell lysis $(1,22,40)$. The present systematic review highlights initial MR images, excluding corticosteroid effects from impacting the findings. Three patterns of neoplastic cell infiltration have been described as diffuse scattered infiltration, perivascular distribution and massive cluster. This could be hypothesized to explain the three contrast-enhancement patterns in diffuse PCNSL. However, in the present systematic review, a corresponding association was not found between contrast-enhancement patterns on neuroimaging and neoplastic cell infiltration patterns on pathology.

Cerebral punctate and curvilinear gadolinium enhancements with FLAIR hyperintensities in the corresponding areas indicate small vessel BBB disruption caused by a direct injury of the endothelial cell (64). Recent studies have shown that T1-weighted dynamic contrast-enhanced and dynamic susceptibility-weighted contrast-enhanced MRI could provide further information about intact microvascular integrity and neoangiogenesis, which can be used to improve the preoperative differentiation of PCNSL and other pathological conditions $(63,65)$.

DWI is useful in distinguishing between PCNSL and other tumors and tumor-mimicking lesions $(1,2,51,57,66,67)$. DWI findings in the present systematic review were isointense, slightly hyperintense and high hyperintense compared with normal gray matter. The extension of DWI hyperintensities corresponded with or without abnormal T2-hyperintensity. It is worth noting that almost all of the articles did not provide or describe ADC maps to distinguish between restricted diffusion and T2 shine-through effect on DWI, as well as cytotoxic and vasogenic edema. As for our present case, a combination of DWI and ADC hyperintense signal means vasogenic edema with T2 shine through effect. Therefore, the diagnostic value of DWI for diffuse PCNL should not be determined depending only on this available literature.

PET/CT is a well-established functional imaging method widely used in clinical neurooncology. In a review published by Kawai et al (68), it was concluded that PET/CT is useful in the diagnosis of typical PCNSL and can differentiate PCNSL from other malignant brain tumors. However, the value of PET/CT appears to be limited in the diagnosis of PCNSL with atypical radiological findings, including disseminated or non-enhancing lesions. PET findings in the present systematic 
review were varied and non-specific. This may be associated with regional cerebral metabolic rates of glucose, cellular differentiation, low cellularity and BBB integrity. The study by Kawai et al $(23,69)$ suggested that the parameters evaluated by FDG kinetic analysis may provide valuable information in the diagnosis of atypical PCNSL. For diffuse PCNSL, the usefulness of whole-body PET scan appears merely for the purposes of excluding systemic lymphoma $(2,26,29,36,66)$.

Rapidly progressing dementia in the elderly and diffuse leukoencephalopathy on MRI should prompt the consideration of diffuse PCNSL. Although a substantial number of studies describe how to differentiate between rapidly progressing dementia in the elderly and diffuse leukoencephalopathy (1-3,2 $6,30,33,34,36,49,51,57,62-71)$, the diagnosis of diffuse PCNSL remains a challenge, and all cases in the present systematic review were misdiagnosed prior to brain biopsy or autopsy. The presumptive diagnosis of diffuse infiltrative brain neoplasia is proposed by the detailed analysis of medical history, clinical presentation, CSF results and serial MRI, including DWI and contrast enhancement. A decision to undertake a brain biopsy as soon as possible may benefit such patients.

The main limitations of the present systematic review are that only a few cases involved DTI (29), MRS $(29,30,49,51)$ or SPECT (49). The diagnostic values of these imaging methods have yet to be investigated in depth. Furthermore, the treatment and prognosis of lymphomatosis cerebri have not been analyzed in detail, as the present study focuses on describing the neuroradiological features of this condition.

In conclusion, although all data in the present systematic review are obtained from case reports, neuroradiological features of lymphomatosis cerebri could be characterized by rapidly progressing dementia in the elderly and diffuse leukoencephalopathy that tends to infiltrate the entire CNS along the corticospinal tract, possible involvement of gray matter, and a slight mass effect and varied contrast-enhancement patterns on MRI. It appears more reasonable to rename the so-called 'lymphomatosis cerebri' as 'diffuse PCNSL' or 'diffuse lymphoma of the CNS'. Non-enhancement or non-mass-like enhancement on MRI may only be a special form of diffuse PCNL during its development and progression. Early brain biopsy will minimize the duration of misdiagnosis in the setting of a presumptive diagnosis of diffuse infiltrative brain neoplasia.

\section{Acknowledgements}

Not applicable.

\section{Funding}

No funding was received.

\section{Availability of data and materials}

All data generated or analyzed during this study are included in this published article. A list of detailed clinical data and neuroradiological findings collected from all available case reports are shown as 'On-line Table I' and 'On-line Table II' in the online repository Baidu Netdisk (https://pan.baidu. com/s/1KeHH0w0aBR6vKO_mLb2lPA).

\section{Authors' contributions}

LL designed the study. LL and RJH searched, collected and amalgamated the literature. RJH and FJ gathered the case data. All authors analyzed and interpreted the data. All authors drafted the manuscript. All authors read and approved final manuscript.

\section{Ethics approval and consent to participate}

Ethical approval for this retrospective study was obtained from the local Institutional Review Board in Guangdong Provincial Corps Hospital of Chinese People's Armed Police Forces, Guangzhou Medical University (Guangzhou, China).

\section{Consent for publication}

Written informed consent was obtained from the daughter of the patient for publication of this case report and any accompanying data.

\section{Competing interests}

The authors declare that they have no competing interests.

\section{References}

1. Hoang-Xuan K, Bessell E, Bromberg J, Hottinger AF, Preusser M, Rudà R, Schlegel U, Siegal T, Soussain C, Abacioglu U, et al: Diagnosis and treatment of primary CNS lymphoma in immunocompetent patients: Guidelines from the European Association for Neuro-Oncology. Lancet Oncol 16: e322-e332, 2015.

2. Bathla G and Hegde A: Lymphomatous involvement of the central nervous system. Clin Radiol 71: 602-609, 2016.

3. Deckert $M$ and Paulus W: Malignant lymphoma. In: WHO Classification of Tumours of the Central Nervous System. 4th edition.Louis DN, Ohgaki H, Wiestler OD and Cavenee WK (eds). IARC Press, Lyon, pp188-196, 2007.

4. Taylor CR and Rudbeck L: Dako education guide: Immunohistochemical staining methods, 6th edition. https://www. agilent.com/cs/library/technicaloverviews/public/08002_ihc_ staining_methods.pdf, 2013.

5. Fuller GN and Kros JM: Gliomatosis cerebri. In: Louis DN, Ohgaki H, Wiestler OD, Cavenee WK (eds). WHO Classification of Tumours of the Central Nervous System, 4th edition. Lyon, France: IARC Press, pp50-52, 2007.

6. Weaver JD, Vinters HV, Koretz B, Xiong Z, Mischel P and Kado D: Lymphomatosis cerebri presenting as rapidly progressive dementia. Neurologist 13: 150-153, 2007.

7. Pandit L, Chickabasaviah Y, Raghothaman A, Mustafa S and Vasudevan A: Lymhomatosis cerebri-a rare cause of leukoencephalopathy. J Neurol Sci 293: 122-124, 2010.

8. Hishikawa N, Niwa H, Hara T, Hara K, Ito M, Shimada S, Yoshida M, Hashizume Y and Murakami N: An autopsy case of lymphomatosis cerebri showing pathological changes of intravascular large B-cell lymphoma in visceral organs. Neuropathology 31: 612-619, 2011.

9. Jain TK, Sharma P, Suman SK, Faizi NA, Bal C and Kumar R: Primary central nervous system lymphoma with lymphomatosis cerebri in an immunocompetent child: MRI and ${ }^{18} \mathrm{~F}-\mathrm{FDG}$ PET-CT findings. Rev Esp Med Nucl Imagen Mol 32: 394-396, 2013.

10. Rivero Sanz E, Torralba Cabeza MÁ, Sanjuán Portugal F and García-Bragado F: Lymphomatosis cerebri mimicking iatrogenic Creutzfeldt-Jakob disease. BMJ Case Rep 2014: pii: bcr2013201246, 2014.

11. Lee PJ, Berrios I, Ionete C and Smith T: Lymphomatosis cerebri: Diagnostic challenges and review of the literature. BMJ Case Rep 2016: pii: bcr2016216591, 2016.

12. Stewart LA, Clarke M, Rovers M, Riley RD, Simmonds M, Stewart G and Tierney JF; PRISMA-IPD Development Group: Preferred reporting items for systematic review and meta-analyses of individual participant data: The PRISMA-IPD statement. JAMA 313: 1657-1665, 2015. 
13. Terae $\mathrm{S}$ and Ogata A: Nonenhancing primary central nervous system lymphoma. Neuroradiology 38: 34-37, 1996.

14. Carlson BA: Rapidly progressive dementia caused by nonenhancing primary lymphoma of the central nervous system. AJNR Am J Neuroradiol 17: 1695-1697, 1996.

15. Wanschitz J, Hainfellner JA, Simonitsch I, Schnizer M, Deisenhammer E, Terunuma $\mathrm{H}$, Iwasaki $\mathrm{Y}$ and Budka $\mathrm{H}$ : Non-HTLV-I associated pleomorphic T-cell lymphoma of the brain mimicking post-vaccinal acute inflammatory demyelination. Neuropathol Appl Neurobiol 23: 43-49. 1997.

16. Furusawa T, Okamoto K, Ito J, Kojima N, Oyanagi K, Tokiguchi S and Sakai K: Primary central nervous system lymphoma presenting as diffuse cerebral infiltration. Radiat Med 16: 137-140, 1998.

17. Bakshi R, Mazziotta JC, Mischel PS, Jahan R, Seligson DB and Vinters HV: Lymphomatosis cerebri presenting as a rapidly progressive dementia: Clinical, neuroimaging and pathologic findings. Dement Geriatr Cogn Disord 10: 152-157, 1999.

18. Thurnher MM, Rieger A, Kleibl-Popov C, Settinek U, Henk C, Haberler C and Schindler E: Primary central nervous system lymphoma in AIDS: A wider spectrum of CT and MRI findings. Neuroradiology 43: 29-35, 2001.

19. Ayuso-Peralta L, Ortí-Pareja M, Zurdo-Hernández M Jiménez-Jiménez FJ, Tejeiro-Martínez J, Ricoy JR, de la Lama A and Bernardo AI: Cerebral lymphoma presenting as a leukoencephalopathy. J Neurol Neurosurg Psychiatry 71: 243-246, 2001.

20. Giglio P, Bakshi R, Block S, Ostrow P and Pullicino PM: Primary central nervous system lymphoma masquerading as herpes encephalitis: Clinical, magnetic resonance imaging, and pathologic findings. Am J Med Sci 323: 59-61, 2002.

21. Moulignier A, Galicier L, Mikol J, Masson H, Molho M and Thiebaut JB: Primary cerebral lymphoma presenting as diffuse leukoencephalopathy. AIDS 17: 1111-1113, 2003.

22. Rollins KE, Kleinschmidt-DeMasters BK, Corboy JR, Damek DM and Filley CM: Lymphomatosis cerebri as a cause of white matter dementia. Hum Pathol 36: 282-290, 2005.

23. Kawai N, Kawanishi M, Tamiya T and Nagao S: Usefulness of $\left[{ }^{18} \mathrm{~F}\right]$ FDG-PET kinetic analysis in non-enhancing primary central nervous system lymphoma: Case report. Ann Nucl Med 19: 415-419, 2005

24. Vital A and Sibon I: A 64-year-old woman with progressive dementia and leukoencephalopathy. Brain Pathol 17: 117-118, 121, 2007.

25. Lewerenz J, Ding X, Matschke J, Schnabel C, Emami P, von Borczyskowski D, Buchert R, Krieger T, de Wit M and Münchau A: Dementia and leukoencephalopathy due to lymphomatosis cerebri. J Neurol Neurosurg Psychiatry 78: 777-778, 2007.

26. Kanai R, Shibuya M, Hata T, Hori M, Hirabayashi K, Terada T and Fujii K: A case of 'lymphomatosis cerebri' diagnosed in an early phase and treated by whole brain radiation: Case report and literature review. J Neurooncol 86: 83-88, 2008.

27. Sugie M,Ishihara K, Kato H, Nakano I and Kawamura M: Primary central nervous system lymphoma initially mimicking lymphomatosis cerebri: An autopsy case report. Neuropathology 29: 704-707, 2009.

28. Clark AJ, Lee K, Broaddus WC, Martin MJ, Ghatak NR, Grossman CE, Baker S Jr and Baykal A: Primary brain T-cell lymphoma of the lymphoblastic type presenting as altered mental status. Acta Neurochir (Wien) 152: 163-168, 2010.

29. Liao MF, Toh CH, Kuo HC, Chu CC, Jung SM and Huang CC: Diffusion tensor images and magnetic resonance spectroscopy in primary central nervous system T-cell lymphoma: A case report. Acta Neurol Taiwan 20: 59-64, 2011.

30. Raz E, Tinelli E, Antonelli M, Canevelli M, Fiorelli M, Bozzao L, Di Piero V and Caramia F: MRI findings in lymphomatosis cerebri: Description of a case and revision of the literature. J Neuroimaging 21: e183-e186, 2011.

31. Leschziner G, Rudge P, Lucas S and Andrews T: Lymphomatosis cerebri presenting as a rapidly progressive dementia with a high methylmalonic acid. J Neurol 258: 1489-1493, 2011.

32. Courtois F, Gille M, Haven F and Hantson P: Lymphomatosis cerebri presenting as a recurrent leukoencephalopathy. Case Rep Neurol 4: 181-186, 2012

33. Keswani A, Bigio E and Grimm S: Lymphomatosis cerebri presenting with orthostatic hypotension, anorexia, and paraparesis. J Neurooncol 109: 581-586, 2012.

34. Kitai R, Hashimoto N, Yamate K, Ikawa M, Yoneda M, Nakajima T, Arishima H, Takeuchi H, Sato K and Kikuta K: Lymphomatosis cerebri: Clinical characteristics, neuroimaging, and pathological findings. Brain Tumor Pathol 29: $47-53,2012$.
35. Yamamoto T, Kojima K, Koibuchi K, Ito S, Higuchi Y, Iwadate Y, Oide T and Kuwabara S: A case of primary central nervous system lymphoma presenting diffuse infiltrative leukoencephalopathy. Intern Med 51: 1103-1106, 2012.

36. Sugino T, Mikami T, Akiyama Y, Wanibuchi M, Hasegawa T and Mikuni N: Primary central nervous system anaplastic large-cell lymphoma mimicking lymphomatosis cerebri. Brain Tumor Pathol 30: 61-65, 2013

37. Sato H, Takahashi Y, Wada M, Shiono Y, Suzuki I, Kohno K, Kato Y, Kawanami T, Sakurada K, Kayama T and Kato T: Lymphomatosis cerebri with intramedullary spinal cord involvement. Intern Med 52: 2561-2565, 2013.

38. Choi CY, Lee CH and Joo M: Lymphomatosis cerebri. J Korean Neurosurg Soc 54: 420-422, 2013

39. Miki Y, Tomiyama M, Kurotaki H, Wakabayashi K and Baba M: Primary central nervous system lymphoma mimicking Bickerstaff's encephalitis. Neurol Sci 35: 139-141, 2014.

40. Giannini C, Dogan A and Salomão DR: CNS lymphoma: A practical diagnostic approach. J Neuropathol Exp Neurol 73: 478-494, 2014

41. Kawakami T, Sakai K, Mimura Y, Senoo Y, Hirabayashi Y, Nakazawa H, Koshihara H, Oguchi K, Takei Y, Ohara S, et al: Development of primary central nervous system lymphoma associated with human immunodeficiency virus and JC virus infection. J Clin Exp Hematop 54: 211-217, 2014.

42. Samani A, Davagnanam I, Cockerell OC, Ramsay A, Patani R and Chataway J: Lymphomatosis cerebri: A treatable cause of rapidly progressive dementia. J Neurol Neurosurg Psychiatry 86: 238-240, 2015

43. Imperiale D, Taraglio S, Atzori C and Testi R: Diffuse leukoencephalopathy due to lymphomatosis cerebri: A clinicopathological report. Neurol Sci 36: 1071-1073, 2015.

44. Meng H, Zhou C, Hao Q, Gao J, Zhang R, Wang Z, Liu Q and Fang S: A rare case of Primary Central Nervous System Lymphoma initially diagnosed as demyelinating encephalopathy. Neuro Endocrinol Lett 36: 124-126, 2015.

45. Hatanpaa KJ, Fuda F, Koduru P, Young K, Lega B and Chen W: Lymphomatosis cerebri: A diagnostic challenge. JAMA Neurol 72: 1066-1067, 2015

46. Chen $\mathrm{H}$ and Dong $\mathrm{H}$ : A rare case of nonenhancing primary central nervous system lymphoma mimic multiple sclerosis. Neurosciences (Riyadh) 20: 380-384, 2015.

47. Hashiguchi S, Momoo T, Murohashi Y, Endo M, Shimamura M, Kawasaki T, Kanada S, Nozawa A, Tada M, Koyano S and Tanaka F: Interleukin 10 level in the cerebrospinal fluid as a possible biomarker for lymphomatosis cerebri. Intern Med 54: 1547-1552, 2015.

48. Izquierdo C, Velasco R, Vidal N, Sánchez JJ, Argyriou AA, Besora S, Graus F and Bruna J: Lymphomatosis cerebri: A rare form of primary central nervous system lymphoma. Analysis of 7 cases and systematic review of the literature. Neuro Oncol 18: 707-715, 2016.

49. Murakami T, Yoshida K, Segawa M, Yoshihara A, Hoshi A, Nakamura K, Ichikawa M, Suzuki O, Yokoyama Y, Toyoshima Y, et al: A case of lymphomatosis cerebri mimicking inflammatory diseases. BMC Neurol 16: 128, 2016.

50. Ge J, Zuo C, Guan Y and Zhao G: Enhanced MRI and ${ }^{18} \mathrm{~F}-\mathrm{FDG}$ PET/CT findings of primary central nervous system lymphoma mimicking encephalitis. Clin Nucl Med 41: e436-e438, 2016.

51. Brandão LA and Castillo M: Lymphomas-part 2. Neuroimaging Clin N Am 26: 537-565, 2016.

52. Fisher D, Mantell BS and Urich H: The clinical diagnosis and treatment of microgliomatosis: Report of a case. J Neurol Neurosurg Psychiatry 32: 474-478, 1969.

53. Schaumburg HH, Plank CR and Adams RD: The reticulum cell sarcoma-microglioma group of brain tumours. A consideration of their clinical features and therapy. Brain 95: 199-212, 1972

54. Tadmor R, Davis KR, Roberson GH and Kleinman GM: Computed tomography in primary malignant lymphoma of the brain. J Comput Assist Tomogr 2: 135-140, 1978.

55. Provinciali L, Signorino M, Ceravolo G and Pasquini U: Onset of primary brain T-lymphoma simulating a progressive leukoencephalopathy. Ital J Neurol Sci 9: 377-381, 1988.

56. Chandana SR, Movva S, Arora M and Singh T: Primary brain tumors in adults. Am Fam Physician 77: 1423-1430, 2008.

57. Sarbu N, Shih RY, Jones RV, Horkayne-Szakaly I, Oleaga L and Smirniotopoulos JG: White matter diseases with radiologic-pathologic correlation. Radiographics 36: 1426-1447, 2016. 
58. Alsherbini K, Beinlich B and Salamat MS: Diffusely infiltrating central nervous system lymphoma involving the brainstem in an immune-competent patient. JAMA Neurol 71: 110-111, 2014.

59. Matsumoto K, Kohmura E, Fujita T, Tsuruzono K, Tsujimura T and Kawano K: Recurrent primary central nervous system lymphoma mimicking neurodegenerative disease-an autopsy case report. Neurol Med Chir (Tokyo) 35: 360-363, 1995.

60. Brecher K, Hochberg FH, Louis DN, de la Monte S and Riskind P. Case report of unusual leukoencephalopathy preceding primary CNS lymphoma. J Neurol Neurosurg Psychiatry 65: 917-920, 1998.

61. Nipanal AV, Madhumathi R, Navya and Angel: CNS lymphoma mimicking a demyelinating lesion. J Evol Med Dent Sci 4: $11555-11559,2015$

62. Smirniotopoulos JG, Murphy FM, Rushing EJ, Rees JH and Schroeder JW: Patterns of contrast enhancement in the brain and meninges. Radiographics 27: 525-551, 2007.

63. Kickingereder P, Sahm F, Wiestler B, Roethke M, Heiland S Schlemmer HP, Wick W, von Deimling A, Bendszus M and Radbruch A: Evaluation of microvascular permeability with dynamic contrast-enhanced MRI for the differentiation of primary CNS lymphoma and glioblastoma: Radiologic-pathologic correlation. AJNR Am J Neuroradiol 35: 1503-1508, 2014.

64. Taieb G, Duran-Peña A, de Chamfleur NM, Moulignier A Thouvenot E, Allou T, Lacour A, Hoang-Xuan K, Pelletier J and Labauge P: Punctate and curvilinear gadolinium enhancing lesions in the brain: A practical approach. Neuroradiology 58: 221-235, 2016

65. da Rocha AJ, Sobreira Guedes BV, da Silveira da Rocha TM, Maia Junior AC and Chiattone CS: Modern techniques of magnetic resonance in the evaluation of primary central nervous system lymphoma: Contributions to the diagnosis and differential diagnosis. Rev Bras Hematol Hemoter 38: 44-54, 2016.
66. Patrick LB and Mohile NA: Advances in primary central nervous system lymphoma. Curr Oncol Rep 17: 60, 2015.

67. Malikova H, Koubska E, Weichet J, Klener J, Rulseh A, Liscak R and Vojtech Z: Can morphological MRI differentiate between primary central nervous system lymphoma and glioblastoma? Cancer Imaging 16: 40, 2016.

68. Kawai N, Miyake K, Yamamoto Y, Nishiyama Y and Tamiya T: ${ }^{18} \mathrm{~F}-\mathrm{FDG}$ PET in the diagnosis and treatment of primary central nervous system lymphoma. Biomed Res Int 2013: 247152, 2013.

69. Kawai N, Okubo S, Miyake K, Maeda Y, Yamamoto Y, Nishiyama Y and Tamiya T: Use of PET in the diagnosis of primary CNS lymphoma in patients with atypical MR findings. Ann Nucl Med 24: 335-343, 2010.

70. Scott BJ, Douglas VC, Tihan T, Rubenstein JL and Josephson SA: A systematic approach to the diagnosis of suspected central nervous system lymphoma. JAMA Neurol 70: 311-319, 2013.

71. Deutsch MB and Mendez MF: Neurocognitive features distinguishing primary central nervous ssystem lymphoma from other possible causes of rapidly progressive sdementia. Cogn Behav Neurol 28: 1-10, 2015

This work is licensed under a Creative Commons Attribution-NonCommercial-NoDerivatives 4.0 International (CC BY-NC-ND 4.0) License. 